\title{
GLAD!
}

Revue sur le langage, le genre, les sexualités

$02 \mid 2017$

Varia

\section{Performer son sexe}

La chirurgie d'amélioration des organes génitaux du début du $\mathrm{XXI}^{\mathrm{e}}$ siècle selon les sites web qui la promeuvent en Suisse romande

Performing Sex/Gender : the Promotion of Contemporary Genital Cosmetic

Surgery on the Web in French-Speaking Switzerland

Hélène Martin, Rebecca Bendjama et Raphaëlle Bessette-Viens

\section{(2) OpenEdition}

\section{Journals}

Édition électronique

URL : http://journals.openedition.org/glad/435

DOI : $10.4000 /$ glad.435

ISSN : 2551-0819

Éditeur

Association GSL

Référence électronique

Hélène Martin, Rebecca Bendjama et Raphaëlle Bessette-Viens, «Performer son sexe », GLAD! [En ligne], 02 | 2017, mis en ligne le 01 juin 2017, consulté le 21 janvier 2021. URL : http:// journals.openedition.org/glad/435; DOI : https://doi.org/10.4000/glad.435

Ce document a été généré automatiquement le 21 janvier 2021.

La revue GLAD! est mise à disposition selon les termes de la Licence Creative Commons Attribution Pas d'Utilisation Commerciale - Pas de Modification 4.0 International. 


\section{Performer son sexe}

La chirurgie d'amélioration des organes génitaux du début du $\mathrm{XXI}^{\mathrm{e}}$ siècle selon les sites web qui la promeuvent en Suisse romande

Performing Sex/Gender : the Promotion of Contemporary Genital Cosmetic

Surgery on the Web in French-Speaking Switzerland

Hélène Martin, Rebecca Bendjama et Raphaëlle Bessette-Viens

\section{Introduction}

Différents sites web de cliniques et cabinets médicaux de Suisse romande proposent désormais des chirurgies d'amélioration des organes génitaux. Aux frontières controversées de l'esthétique et de la reconstruction, cette chirurgie n'est ni « obligatoire ni indispensable d'un point de vue organique ou fonctionnel » (Guirimand 2005 : 73). Les interventions sont donc envisagées en fonction d'un choix personnel, l'aval du médecin étant toutefois nécessaire pour qu'elles aient lieu, comme cela est relevé sur l'un des sites qui en font la promotion:

Après une discussion approfondie avec le patient permettant de mieux comprendre ses envies et besoins, les médecins et chirurgiens esthétiques définiront les objectifs

à atteindre et les soins les plus adaptés, en matière de correction de contours corporels harmonieux, embellissement de votre peau ou programmes de rajeunissement ${ }^{1}$.

2 Ces chirurgies travaillent les organes génitaux de sorte, par exemple, à ce que les petites lèvres soient symétriques, la taille des grandes lèvres augmentée, le mont de Vénus réduit, le vagin resserré, l'hymen ou le clitoris reconstruit, le pénis élargi.

Puisque l'internet se présente comme "un média de la vie de tous les jours » (OFS, 2015), particulièrement fréquenté par les internautes qui se questionnent sur la santé et la sexualité (OFS 2015), nous avons choisi d'analyser ces sites en tant que discours. Nous nous demandons comment ces derniers procèdent pour motiver le recours aux interventions chirurgicales d'amélioration des organes génitaux. Les sites web de cliniques et cabinets médicaux de Suisse romande constituent un corpus parmi d'autres que nous analysons dans le cadre d'une recherche ${ }^{2}$ actuellement en cours. Celle-ci 
propose d'explorer les liens établis entre, d'une part, la morphologie et la fonctionnalité des organes génitaux et, d'autre part, les conceptions de la sexualité et de l'identité sexuée.

4 Envisageant que le sexe et le genre sont mutuellement constitutifs (Delphy 2001; Fausto-Sterling \& Touraille 2014), nous considérons que la chirurgie d'amélioration des organes génitaux permet d'étudier la sexuation du biologique et du social (Gardey 2013 ; Jaunait et al. 2014). Nous nous inscrivons ainsi dans une approche située, pour laquelle la science produit des corps sexués non seulement en fonction d'une posture mais également en fonction de pratiques et de techniques, par exemple hormonales ou chirurgicales (Haraway 1988, 1991; Lynda Birke 1997; Schiebinger 2004; Harding 1991). Une telle approche anti-essentialiste permet de "désubstantialiser les objets sociaux » (Pfefferkorn 2012: 23), par exemple, et pour ceux qui nous intéressent, les catégories sociales de sexe et de sexualité. De notre point de vue constructiviste, ces catégories résultent en effet de rapports sociaux, qui les constituent dans leurs aspects idéels et matériels (Kergoat 2009), les situant les unes par rapport aux autres en fonction de processus de distinction et de hiérarchisation (Delphy 2001). Ces rapports sociaux sont donc également des rapports de domination. C'est l'articulation de ces rapports de domination tels qu'ils sont véhiculés sur les sites web de cliniques et de cabinets médicaux que nous nous proposons de saisir afin de comprendre comment ils constituent la sexualité et l'identité sexuée (Bajos, Ferrand \& Andro 2008).

Nous montrons que, à sa manière, le discours étudié condamne des contraintes qui pèseraient sur la sexualité des femmes, des contraintes qu'il attribue, pour certaines d'entre elles, à des pressions d'ordre culturel, tandis qu'il en naturalise d'autres. Le discours étudié présente la chirurgie esthétique des organes génitaux comme une manière de triompher individuellement de ces contraintes, tout en reproduisant des normes de genre et de sexualité. Ce faisant, et c'est ce que nous nous attachons à démontrer, le discours étudié tend à invisibiliser les normes de genre et de sexualité en les faisant passer pour des choix individuels.

Dans la première partie de cet article, nous situons la chirurgie que nous étudions dans son contexte d'émergence, en revenant sur la médicalisation de la sexualité et, dans ce cadre, sur différents changements d'appréhension du sexe et de la sexualité qui ont conduit à l'apparition d'interventions chirurgicales sur les organes génitaux en vue de normaliser la sexualité (partie 1). Nous qualifions ensuite le discours analysé (partie 2), avant d'en proposer une analyse basée sur le repérage des procédés discursifs dont il use et des champs lexicaux auxquels il renvoie (partie 3). Nous développons cette analyse autour de trois thèmes qui constituent selon nous les ressorts du discours étudié : la promotion de l'épanouissement sexuel (3.1), les entraves morphologiques (3.2) et les contraintes culturelles (3.3). Dans la quatrième et dernière partie, nous proposons une mise en perspective de la manière dont ce discours définit le sexe (morphologique et identitaire) et la sexualité et se situe dans son contexte (partie 4), d'une part vis-à-vis de ce qu'il présente comme des défauts du corps (4.1) et d'autre part en regard du devoir de production de soi, que nous analysons en termes d'individualisation des rapports sociaux (4.2.). 


\section{L'émergence de la chirurgie esthétique sur les organes génitaux}

7 Les pratiques d'amélioration des organes génitaux auxquelles nous nous intéressons s'inscrivent dans un cadre d'appréhension médicale de la sexualité, qui émerge au XIX siècle. Ce cadre extrait la sexualité de la morale religieuse et en fait un objet des discours scientifiques, qui la produisent comme un domaine autonome (Foucault 1976; Giami 2007). Différentes représentations du sexe et de la sexualité vont se superposer et se succéder jusqu'à nos jours et déterminer l'attribution de qualités aux organes génitaux associés à la sexualité. En tant que discipline médicale, la chirurgie va travailler la morphologie génitale en fonction des normes qu'elle établit et des techniques dont elle dispose.

Dans le contexte colonial et évolutionniste du xix siècle, les scientifiques nourrissent un intérêt particulier pour les sexes jugés hypertrophiés de femmes européennes de milieux modestes ou déviants (Chaperon $2008: 27$ ) et de femmes et d'hommes racisés. Ces morphologies sont associées à une sexualité débridée opposée à une sexualité ordonnée par la civilisation (Gilman 1985; Halberstam 1998; Nurka \& Jones 2013; Peiretti-Courtis 2015 ; Taraud 2011). Tantôt, ce qui est évalué comme hypertrophié est considéré comme provoqué par des comportements sexuels excessifs ${ }^{3}$; tantôt, c'est la particularité morphologique qui est pensée comme la cause d'une sexualité jugée excessive. Ces observations justifient des pratiques comme l'excision rituelle qui a lieu dans certains pays d'Afrique et, en Occident, elles entraînent des pratiques correctrices médicalisées. En Angleterre puis en Amérique du Nord au début du $\mathrm{xx}^{\mathrm{e}}$ siècle, par exemple, la clitoridectomie est utilisée pour traiter la nymphomanie (Perrot 1998 ; Scull \& Favreau 1987). La castration masculine curative est réalisée chez des délinquants sexuels aux États-Unis, en Suisse, en Allemagne et au Danemark (Erlich 2007) jusqu'au début $\mathrm{du} \mathrm{xx}^{\mathrm{e}}$ siècle. La castration féminine est effectuée chez des femmes présentant des «affections nerveuses ou présumées symptomatiques des ovaires (névralgies, dysménorrhée, hystérie)»(Erlich $2007: 183$ ), dans des proportions qui augmentent avec les politiques eugénistes (en particulier aux États-Unis, en Allemagne et en Suisse). Toutefois, avec la fin des empires coloniaux et suite à leur utilisation par le régime nazi, les théories raciologiques et eugénistes sont peu à peu désavouées, puis abandonnées. L'idée de particularités morphologiques liées aux races et aux groupes déviants est réfutée au profit de l'argument de la variété individuelle des morphologies sexuelles (Peiretti-Courtis 2015).

$9 \mathrm{Au} \mathrm{xx}$ e siècle, la médecine a passé d'une centration sur les dangers pour la vie à un intérêt plus large visant à son amélioration (Vigarello 2004): alors que la santé équivalait à l'absence de maladie au XIX ${ }^{\mathrm{e}}$ siècle, elle s'est étendue au cours du xxe siècle à un état de bien-être général et est devenue "un "processus" devant être maintenu, conquis et développé par différentes méthodes »(Giami 2007 : 56) ${ }^{4}$. La sexualité ellemême s'éloigne d'une centration sur la reproduction pour devenir une activité associée au bien-être et à l'épanouissement de l'individu. Au cours de la première moitié du $\mathrm{xx}^{\mathrm{e}}$ siècle, la recherche de plaisir sexuel (dans le cadre du mariage) devient une norme, tout en demeurant spécifique à chaque sexe (Chaperon 2004 ; Bozon 2013). À la norme du plaisir partagé s'opposent alors la chasteté et l'abstinence, l'éjaculation précoce ou la frigidité (Chaperon 2002). Ces nouveaux troubles du plaisir donnent lieu à certaines interventions chirurgicales sur les organes génitaux, par exemple l'opération, promue 
par Marie Bonaparte, visant à rapprocher le clitoris du vagin de sorte à «faciliter la migration interne de l'orgasme" (Chaperon 2002: 104) ou l'ablation du prépuce clitoridien comme remède à la frigidité (Erlich 2007 ; Gilman 1997).

10 Cependant, la référence à la morphologie pour expliquer les troubles du plaisir s'atténue avec l'émergence d'un nouveau cadre d'appréhension de la sexualité dès la moitié du $\mathrm{xx}^{\mathrm{e}}$ siècle. Sur la base de leur étude en laboratoire de centaines de couples pratiquant une activité sexuelle, Masters et Johnson produisent la théorie du cycle normal de la sexualité. Ce cycle s'articule, tant chez les hommes que chez les femmes, dans les quatre phases, similaires sur le plan physiologique, de l'excitation, du plateau, de l'orgasme et de la résolution. Ce modèle fonctionnaliste envisage la sexualité comme étant régie par des lois physiologiques neutres du point de vue du sexe et les différences observées dans la pratique sexuelle des hommes et des femmes sont renvoyées à la socialisation (Chaperon 2010 ; Gardey \& Hasdeu 2015). En fonction de ce modèle, qui fait autorité jusque dans les années $1980^{5}$, les déviances au cycle normal de la sexualité sont l'anorgasmie, le vaginisme, l'éjaculation précoce ou retardée, la baisse du désir, etc. Acquises, elles donnent lieu à des thérapies comportementales (Bozon 2013 ; Gardey \& Hasdeu 2015).

11 L'appréhension de la neutralité des processus physiologiques de la sexualité et le renvoi des différences de comportements sexuels à la socialisation ne remettent toutefois pas en question le modèle du dimorphisme sexuel. C'est ainsi que dans la diversité désormais reconnue des morphologies sexuelles, celles qui ne sont pas clairement lisibles du point de vue du dimorphisme sexuel sont pathologisées, que la morphologie génitale paraisse ambiguë ou que certains critères du sexe ne se superposent pas (par exemple la présence d'un utérus chez un homme ou non-cohérence entre le phénotype et le génotype). Dans un contexte de développement des techniques de la chirurgie plastique (en particulier suite à la Seconde Guerre mondiale), de la généralisation des antibiotiques (Löwy 2003: 85) et de la production d'hormones artificielles, des interventions chirurgicales sont réalisées sur des individus en bonne santé mais dont le sexe ne paraît pas fini (Fausto-Sterling 1999) : plus ou moins invasives, elles visent à rectifier la nature en produisant des sexes conformes au modèle du dimorphisme sexuel et de l'hétérosexualité pénétrative. Il s'agit par exemple de raccourcir un clitoris jugé trop court pour être un pénis, d'augmenter la taille d'un micropénis, de produire artificiellement un vagin, de corriger des hypospadias ou des prépuces dits incomplets (Boillot, Teklali, Moog \& Droupy 2013). Dans le même contexte se développe l'offre pour les chirurgies de changement de sexe, qui avait timidement débuté dans les années 1920-1930 (Erlich 2007). En effet, s'il n'est pas possible d'agir sur la cause des troubles de l'identité sexuelle, tout au moins la médecine peut-elle agir sur la morphologie (Macé 2010). Ces interventions corrigent la morphologie pour la rendre lisible et congruente avec l'identification de sexe; elles témoignent d'une dissociation idéologique du sexe en deux qualités : le sexe biologique issu des théories naturalistes du XIX ${ }^{e}$ siècle et l'identification de sexe (ou le genre), qui devrait lui correspondre.

Réalisées de manière massive dans les pays occidentaux depuis les années 1950, les chirurgies de (ré)assignation du sexe sont désormais l'objet de contestations, portées par différents mouvements sociaux: les mouvements de défense des droits des personnes intersexes, par exemple, associent les opérations d'assignation sexuelle à des mutilations et revendiquent le droit des individus de choisir de recourir ou non aux traitements médicaux, tandis que des mouvements transgenres revendiquent le droit 
de ne pas choisir son sexe (Chaperon 2010; Macé 2010; Martin 2014). Cette contestation traduit une mise en question de l'argument naturaliste, selon lequel le sexe biologique (quels que soient les critères retenus : génotype, phénotype, hormonal, etc.) est la cause de l'identité de sexe ou, tout au moins, de la légitimité de la médecine d'assigner un sexe aux individus.

De telles prises de positions se développent dans un contexte qui valorise le bien-être et la responsabilité individuelle dans les questions de santé. Ces nouvelles normes, loin d'émanciper ou de libérer le sujet des contraintes sociales, consistent plutôt dans un déplacement de ces dernières : le sujet est conduit à prendre en charge, de manière volontaire, sa propre normalisation (Bozon 2009; Martin, Hertz \& Rey 2015). Cette tendance à l'individualisation des comportements s'exprime également dans l'appréhension de la sexualité. À partir du dernier tiers du xxe siècle, l'épanouissement sexuel est institué comme une condition de bonne santé, ainsi qu'en témoigne l'émergence du concept de santé sexuelle ${ }^{6}$. La sexualité de plaisir, distincte de la procréation pour les hommes comme pour les femmes, devient une dimension centrale dans la définition de soi, si bien que les individus qui ne témoignent pas d'une vie sexuelle satisfaisante paraissent incomplets (Bozon 2013). Les conditions technologiques ${ }^{7}$ et idéologiques sont réunies pour que la chirurgie plastique se mette à travailler les organes génitaux, proposant des interventions non nécessaires visant à améliorer le sexe et la sexualité. Cette chirurgie relègue à ses frontières les interventions sur des organes considérés comme pathologiques. Toutefois, et comme en témoignent les polémiques sur la légitimité des interventions de (ré)assignation sexuelle, l'établissement d'une définition des morphologies pathologiques ainsi que, en conséquence, de frontières entre différentes chirurgies plastiques (esthétique, réparatrice, correctrice, etc.) fait, comme elle l'a d'ailleurs toujours fait (Guirimand 2005), l'objet de polémiques.

Le chirurgien Michael P. Goodman (2009) situe le premier article scientifique portant sur la labioplastie en $1984^{8}$, mais cette chirurgie se développe surtout, dans les pays industrialisés, depuis les années 1990 (Foldès, Droupy \& Cuzon 2013). Dans les médias états-uniens, la chirurgie esthétique des organes génitaux féminins apparaît pour la première fois en 1998, quand deux chirurgiens de Los Angeles, Gary Atler et David Matlock, publicisent certaines interventions telles que la nymphoplastie, la vaginoplastie, la réduction du capuchon clitoridien et les injections d'acide dans le point $G$ en les présentant comme des pratiques d'embellissement du sexe et d'amélioration de la sexualité (Tiefer 2008). En France, l'anthropologue Michel Erlich (2007 : 186) date la médiatisation de la "nouvelle chirurgie sexuelle » à l'année 1994, lorsque deux articles sont publiés à son sujet. Par nouvelle chirurgie sexuelle, Erlich (2007: 180) entend une "chirurgie de l'apparence et du plaisir", qui investit la morphologie génitale au nom d'une sexualité de plaisir. La chirurgie des organes génitaux est ainsi passée en un peu plus d'un siècle « du domaine de la répression du plaisir à celui de son épanouissement » (Erlich 2007 : 180).

15 Tout comme les prothèses, l'amélioration cognitive ou génétique, ou encore d'autres interventions cosmétiques, les chirurgies esthétiques des organes génitaux contemporaines visent à l'amélioration de certaines caractéristiques ou fonctions humaines qui vont au-delà de ce qui est nécessaire à la vie ou à la réparation du corps : comme nous le verrons, elles associent à la modification esthétique une amélioration des capacités sexuelles et sexuées, si bien qu'elles relèvent du développement des 
technologies d'amélioration ou de transformation du corps (Hogle 2005 ; Grino 2014). Ces technologies, qui transforment la matérialité du corps et la performance des individus, illustrent qu'à l'issue du XIX ${ }^{\mathrm{e}}$ siècle a succédé à l'idée de la rigidité du sexe celle d'une "plasticité technologique du genre» (Preciado 2005: 67). Mises au "service" des individus tenus de prendre en charge leur propre identification de genre, ces technologies participent à la production des identités sociales de sexe et de la sexualité :

Dans ce temps qui nous est contemporain des relations entre genre, corps et biomédecine, il est de la responsabilité des individus de s'appuyer sur des «ressources » ou des «solutions techniques et médicales » [...] qui redéfinissent normativement et de façon puissante ce que le social (et les rapports de genre qui y ont cours) peut et doit être. (Gardey $2013: 149-150$ )

C'est bien en tant qu'instrument de production des organes sexuels, de la sexualité et de l'identité de genre que nous comprenons la chirurgie d'amélioration des organes génitaux sur les sites que nous avons étudiés.

\section{Le discours des sites web qui promeuvent la chirurgie d'amélioration des organes génitaux}

17 Le discours des sites web des cliniques et cabinets médicaux proposant des chirurgies d'amélioration des organes génitaux s'inscrit tout d'abord dans un registre médical. Ces sites affichent en effet une fonction informative dans le domaine de la santé sexuelle: ils décrivent les morphologies jugées problématiques, expliquant les causes et les conséquences des particularités morphologiques susceptibles d'une amélioration et proposent des prises en charge dans le cadre d'une clinique ou d'un cabinet certifiés. Les entretiens avec des médecins (gynécologues et chirurgien-nes, en cabinet et en clinique) proposant ces chirurgies nous ont conduites à constater que le contenu des sites est souvent construit et alimenté par les médecins eux-mêmes ${ }^{9}$. Toutefois, le discours de sites web de cliniques et cabinets présente également une fonction économique : ces sites ont pour but de promouvoir ces interventions et, en ce sens, le discours qu'ils véhiculent est également publicitaire ${ }^{10}$. Précisons toutefois que la publicité médicale est régie par le Code de déontologie de la Fédération des médecins suisses (FMH) qui impose une publicité « objective », "fondée sur l'expérience » et qui "répond aux besoins des patientes et confrères ${ }^{11}$ ». Or, pour vendre, le discours des sites web doit plaire, et notamment « se caler aux représentations, tendances et modes sociales, aux désirs et aspirations des individus» (Sacriste 2001: 493). La littérature portant sur le discours publicitaire met en évidence un certain nombre de procédés discursifs, repérables dans des traces linguistiques, auxquels ce discours recourt. Parmi eux, nous retenons d'une part la typification de représentations et de valeurs sociales, qui sont présentées sous la forme raccourcie de stéréotypes ou d'archétypes et qui apparaissent d'ailleurs comme tels au public (Sacriste 2001: 495); et, d'autre part, la redondance et l'usage d'énoncés gnomiques, à savoir l'affirmation en tant que vérités générales de croyances stéréotypées (Pahud 2006 : 154). Le corpus de sites web se situe donc au carrefour du médical - des médecins informent sur différentes anomalies organiques et troubles de la sexualité en fonction d'un lexique médical spécialisé - et du publicitaire - il vise, dans le cadre du Code de déontologie de la FMH, la promotion des interventions chirurgicales d'amélioration des organes génitaux. 
18 Partant du postulat que, « loin d'afficher la cohérence d'un discours doté de sa propre rationalité, le discours publicitaire constitue, comme la plupart des discours sociaux, un espace de prises de position et de significations tout à fait disparate mais hautement significatif » (Soulages 2004 : 52), nous nous intéressons à la manière dont ce discours construit les organes génitaux et la sexualité, dans l'objectif de comprendre comment il articule morphologie et identité sexuée. Nous avons soumis nos données à une analyse thématique via le logiciel de traitement de données qualitatives NVivo. Nous avons classé les données en fonction de trois thèmes : 1) le contenu formel de cette chirurgie : quelles interventions sont proposées? 2) les visées générales de cette chirurgie : que propose-t-elle et soutient-elle en matière de sexe et de sexualité? 3) les caractéristiques du sexe qui constituent des raisons d'interventions chirurgicales: quelles sont les morphologies susceptibles d'une opération et pourquoi?

\section{Les ressorts du discours étudié}

Notre corpus est constitué de vingt-huit pages issues de onze sites web de cliniques et cabinets de Suisse romande, consultées en octobre 2014 et avril et mai $2015^{12}$. Les sites web des cliniques et cabinets médicaux constituent l'un des deux corpus issus du web que nous étudions. Pour constituer ce corpus virtuel, nous avons exploré le web à la manière d'internautes qui $\mathrm{y}$ chercheraient des informations sur la chirurgie d'amélioration des organes génitaux : nous avons fait une recherche Google avec les mots clés "Suisse romande » + « chirurgies esthétique » ou " chirurgie cosmétique » ou les noms des interventions ("nymphoplastie», " pénoplastie», etc.), ce qui nous a conduites aux onze sites étudiés. Afin d'écarter les erreurs induites par les cookies, nous avons pris soin d'effacer l'historique et le stockage des cookies. Toutefois, les sites référencés sur Google arrivent toujours dans les premiers résultats avec les mêmes mots-clés. Malgré ce point, ou peut-être par sa présence, la procédure utilisée est très proche de celle employée par une personne recherchant sur le web des informations sur la chirurgie des organes génitaux, ou sur l'une d'entre elles, réalisée en Suisse romande. Nous avons donc procédé par mise en situation, si bien que les résultats que nous proposons sont situés et non pas basés sur un matériel nécessairement exhaustif.

Les sites répertoriés présentent les interventions réalisées par des gynécologues et des chirugien-nes exerçant soit en cabinet privé soit en clinique privée. Chaque site propose une palette d'interventions, plus ou moins nombreuses, et réunies sous différentes appellations, "chirurgie intime» ou "des zones intimes", chirurgie plastique ou reconstructive "gynécologique ", "vaginale » ou " génitale », qu'il présente sur une à douze sections, qui sont accessibles en un à quatre clics. Par exemple, lors de notre netnographie (octobre 2014 à avril et mai 2015), le site centrefeelwell.ch amène en trois clics à "chirurgie intime" (Chirurgie plastique esthétique > Corps et extrémités > Chirurgie intime), une page sur laquelle il présente la nymphoplastie et la pénoplastie ; ou encore, le site medfemme.com, permet d'accéder à une description de chacune des douze interventions proposées (un clic par intervention). De plus, chaque site organise ainsi son discours de manière originale (noms et nombre des interventions, types d'informations données, par exemple sur les risques ou les suites de l'opération, présence ou non d'une FAQ, etc.), mais on observe un certain nombre de traits récurrents. Tous les sites analysés promeuvent des interventions sur les organes génitaux féminins : le clitoris, le vagin, le pubis, les petites et les grandes lèvres (la 
nymphoplastie, ou labioplastie, est proposée sur tous les sites), l'hymen et même le point $G$ peuvent être travaillés. En revanche, les interventions sur les organes génitaux masculins sont peu promues: seuls trois sites en mentionnent. Ces dernières interventions concernent toutes le pénis, qu'il s'agit d'allonger, d'élargir ou de circoncire. Cette relative absence de discours sur les organes génitaux masculins illustre les constats sociologiques que la chirurgie esthétique s'adresse surtout aux femmes en raison de leur assignation au devoir de beauté (Wolf 1990 ; Davis 1995 ; Le Hénaff 2013). Si toutes les femmes sont concernées par des standards de beauté, elles ne sont toutefois pas égales face à des normes dominantes de beauté. Les sites étudiés s'adressent, de plus, à des personnes cisgenres, dont l'appartenance sexuelle est médicalement claire: il n'est fait aucune mention de chirurgie esthétique sur des personnes diagnostiquées intersexes ou comme présentant une dysphorie de genre ${ }^{13}$, pour lesquelles l'information se trouve dans des sites ou des rubriques distinctes ("variations du développement sexuel ${ }^{14}$ », " chirurgies de réassignation sexuelle ${ }^{15}$ ", " chirurgie transgenre ${ }^{16}$ ») - même si certains noms d'interventions sont employés sur différents sites et rubriques (par exemple "vaginoplastie» désigne à la fois des interventions destinées à resserrer le vagin sur les sites et rubriques consacrés à l'amélioration des organes génitaux et des constructions de vagin sur des sites et rubriques adressées aux personnes transgenres). Il faut relever ici la proximité non seulement technologique mais aussi lexicale de ces différentes interventions qui, toutes, peuvent être comprises comme des technologies du genre - les distinctions entre amélioration des organes génitaux, assignation et réassignation sexuelle ainsi que les définitions du normal et pathologique relevant de normes sociales et médicales en vigueur. Enfin, tous les sites organisent leur argumentation autour de l'accès au bienêtre et à l'épanouissement sexuel. Cette observation constitue l'amorce et le fil rouge de notre analyse.

\section{La promotion de l'épanouissement sexuel}

21 Par la répétition d'énoncés présentés comme des vérités générales - en d'autres termes des énoncés gnomiques -, les sites promouvant la chirurgie d'amélioration du sexe présentent de très nettes redondances en ce qui concerne leurs visées: les interventions proposées permettraient le bien-être et l'épanouissement sexuel, faisant ainsi écho à l'importance de l'esthétique et à la centralité de la sexualité dans la définition de soi. Le discours affirme ainsi qu'(1) «il est légitime de vouloir se sentir mieux », que (2) « nul ne souhaite vieillir et voir décliner le fonctionnement de l'une ou l'autre partie de son corps. " Aussi, les chirurgies proposées permettent (3) «de retrouver un confort et surtout de ne plus avoir de gêne ou de douleurs lors des rapports sexuels » en vertu du constat que :

(4) [À] l'heure actuelle et grâce à la chirurgie reconstructive, aucune femme ni aucun homme n'est plus contraint de supporter une déficience corporelle psychiquement pesante ou qui causerait des douleurs.

Les sites web référencés désignent dès lors la chirurgie d'amélioration des organes génitaux comme l'alliée de celles et ceux qui se sentent mal dans leur corps et dans leur sexualité. (5) «Les femmes [...] souhaitent que leur gynécologue les écoute et leur offre des solutions viables ", lit-on par exemple sur le site d'un cabinet gynécologique, tandis que, dans un même esprit, une clinique spécialisée en chirurgie plastique affirme : 
(6) $[\mathrm{N}]$ ous, chirurgiens plasticiens, [...] savons comment on peut se sentir mal psychiquement et perdre en qualité de vie lorsqu'on ne peut confier son malêtre et ses problèmes à une personne de confiance. permet de mettre au jour deux types d'entraves qui ne sont pas exclusives l'une de l'autre: les «anomalies » morphologiques, liées aux particularités individuelles et à l'expérience de vie, et les contraintes culturelles, qui prennent la forme de pressions d'ordre symbolique ou matériel sur la sexualité.

\section{Les anomalies morphologiques}

Une partie des interventions promues sur les sites web des cliniques et des cabinets médicaux est motivée par des causes morphologiques. Les sites mettent en discours des défauts innés et acquis sur le parcours de vie, invoquant par exemple des (7) « anomalies morphologiques » relevant du (8) «patrimoine génétique » ou encore des conséquences ordinaires de l'accouchement ou le vieillissement.

L'opération la plus fréquemment citée dans ce registre est la nymphoplastie. Cette dernière répond à une (9) «hypertrophie » des petites lèvres - une (10) «pathologie peu connue » - que ces dernières soient (11) «en excès ", (12) «trop grandes, ou asymétriques », (13) «détendues » ou (14) «relâchées", (15) «trop larges ou asymétriques » :

(16) Les petites lèvres débordent des grandes lèvres lorsque ces dernières sont peu épaisses, ou présentent un aspect plissé, «fripés ». De nombreuses femmes sont psychologiquement affectées. Elles craignent le regard du partenaire sexuel ou que ces lèvres hypertrophiées se laissent deviner sous un vêtement serré [...].

Des interventions sont également proposées sur les grandes lèvres, (17) «qui peuvent devenir atrophiques, c'est-à-dire perdre de leur épaisseur et leur côté charnu » et qu'il s'agit cette fois d'augmenter de sorte à dissimuler les petites lèvres. L'intervention consiste alors en une (18) «plastie labiale de grossissement » ou en une (19) « injection de graisse ». D'autres interventions réduisent le volume du pubis, qui peut présenter une (20) «masse graisseuse excédentaire ».

Si ces particularités morphologiques sont traitées, c'est qu'elles sont comprises comme pouvant engendrer différentes gênes mécaniques et psychologiques, les unes et les autres étant considérées comme entravant le plein épanouissement sexuel ou personnel des femmes. La nymphoplastie par exemple (21) « réduira significativement la gêne des patientes et augmentera leur confiance en soi » et elle est proposée pour faciliter l'accès au plaisir lors de relations sexuelles et pallier à différents empêchements dans la réalisation d'activités ordinaires comme faire du vélo ou se vêtir d'un legging :

(22) Si l'aspect esthétique est important, de nombreuses patientes sollicitent le chirurgien pour une nymphoplastie en raison de douleurs lors des rapports (dyspareunies), des difficultés pour s'habiller (port de sousvêtement, string) et gênes lors de la pratique du sport. 
D'autres défaillances sexuelles justifiant une intervention chirurgicale relèvent du parcours de vie. De manière générale, le vieillissement est présenté comme un facteur affectant le sexe et la sexualité des femmes. Dans le parcours de vie des femmes, un événement en particulier est présenté comme néfaste pour leur sexualité, l'accouchement :

(23) Après un accouchement ou même par le seul effet du vieillissement, le vagin peut se détendre et les muscles qui l'entourent peuvent perdre force et tonicité. [...] En réduisant le diamètre du vagin, la plastie vaginale permet une augmentation des frictions durant la pénétration, rendant l'acte bien plus agréable pour la patiente comme pour son partenaire.

Les épisiotomies ${ }^{17}$ réalisées lors de l'accouchement peuvent de plus laisser des (24) "cicatrices épaisses et douloureuses, douleurs qui s'accentuent lors des rapports sexuels » sur lesquelles la chirurgie peut intervenir en vue de leur atténuation. L'épisiotomie est présentée comme une conséquence nécessaire de situations d'accouchement et, en tant que telle, elle n'est pas associée à une pratique culturelle mais à un acte chirurgical nécessaire ; mais le discours des sites web analysés la définit également comme une pratique qui peut être corrigée et, à cet égard, elle est conjointement associée au lexique de la culture, comme nous le verrons.

Soutenant l'épanouissement sexuel et le bien-être, le discours analysé réfute ainsi que (25) « les sollicitations, nobles mais parfois traumatisantes, qu'impose l'accouchement sur les organes génitaux féminins » affectent fatalement et durablement la sexualité des femmes :

(26) Les femmes qui ont eu des enfants souhaitent une solution pour rajeunir leur vagin et jouir de la meilleure expérience sexuelle possible.

D'autres circonstances du parcours de vie peuvent affecter les organes génitaux féminins et justifier une intervention, comme un changement de poids :

(27) Après un amaigrissement, certaines femmes voient leurs grandes lèvres diminuer de volume et ne plus recouvrir les petites lèvres.

32 La nymphoplastie, l'augmentation ou la réduction du volume des grandes lèvres, la réduction du pubis, la vaginoplastie, la réparation des épisiotomies répondent donc à des défauts innés ou acquis des organes génitaux féminins, dans l'objectif d'augmenter le bien-être sexuel des femmes.

Les organes génitaux masculins sont également susceptibles de bénéficier d'une intervention de chirurgie esthétique :

(28) Les hommes qui présentent un pénis de taille réduite peuvent aussi bénéficier d'une pénoplastie d'allongement ou d'augmentation.

34 Toutefois, selon le discours des quelques sites web analysés qui en font mention, cette intervention ne participerait pas à l'amélioration de l'érection des patients et n'aurait donc pas d'incidence mécanique sur la sexualité des hommes. Pour cette raison, cette chirurgie est parfois désavouée en même temps qu'elle est présentée : 
(29) Cette chirurgie intime chez l'homme n'améliore pas la fonction, mais uniquement l'aspect visuel. Cette technique de chirurgie intime n'est pas pratiquée par le $\operatorname{Dr}[\mathrm{X}]$.

(30) La phalloplastie (section du ligament suspenseur, greffe de graisse, injection d'acide hyaluronique) modifie essentiellement le pénis au repos et son succès éventuel repose sur une bonne évaluation psychologique et sexologique préalable.

Évoquer une évaluation psychologique laisse entendre que les demandes des hommes sont peu légitimes, éventuellement associées à une instabilité psychologique. Quant au raccourcissement du prépuce pour raison esthétique, il est proposé sur un site mais ne donne lieu à aucun commentaire. Les données sur les chirurgies des organes génitaux masculins ne sont pas suffisantes pour formuler des analyses ayant une portée générale. Cependant, la manière dont elles sont (peu) présentées sur les sites analysés va dans le sens des analyses de Yannick Le Hénaff (2013) et de Kathy Davis (2002) : l'accès des hommes aux interventions de chirurgie esthétique est limité et controversé, notamment parce qu'y recourir transgresse des normes dominantes de la masculinité comme celles ne pas trop se soucier de son apparence et de ne pas perdre le contrôle de son corps - par exemple sur la table d'opération (Davis 2002). Enfin, dans le cas étudié, la légitimité des femmes à recourir aux chirurgies d'amélioration des organes génitaux est également due, pour paraphraser Le Hénaff (2013:20), à "l'étiquetage pathologique » qui est lié au sexe féminin. Témoignent de cet étiquetage pathologique la variété des dysfonctionnements innés et acquis qui toucheraient les femmes et l'inscription des interventions promues dans une zone floue entre normalité et pathologie; les défaillances traitées sont en effet référées à des caractéristiques morphologiques féminines normales mais problématiques, ainsi qu'au parcours de vie des femmes présenté comme ordinaire mais potentiellement traumatisant sur le plan sexuel.

Enfin, on ne l'aura pas manqué, le discours des sites web analysés reproduit, et même exagère, le dimorphisme sexuel en réalisant des interventions qui tendent à augmenter ce qu'il définit comme des différences morphologiques. Les interventions visent surtout à réduire le sexe féminin, aux deux sens de le rendre plus petit et plus intérieur : elles façonnent un sexe féminin dont les petites lèvres sont cachées par les grandes lèvres, qui ne doivent être ni trop grandes (le sexe doit rester petit) ni trop petites (ne pas laisser paraître des petites lèvres). En bref, le féminin est idéalement intérieur, pour ne pas dire intériorisé. Il est également jeune, comme l'illustre la présence sur huit sites d'une intervention de "rajeunissement» vaginal. Cette visée ne concerne pas seulement l'apparence du sexe mais aussi la performance sexuelle - une performance qui paraît du coup être le privilège de la jeunesse, et qui est opposée à la maternité. La modestie du discours consacré aux organes génitaux masculins traduit l'idée d'une performance masculine allant de soi ou du moins distincte de l'esthétique, et les rares interventions proposées visent à exprimer l'extériorité et la puissance masculine que figure un pénis large, long et fonctionnel dans le cadre de la pénétration. Enfin, les femmes et les hommes sont présentés comme des catégories évidentes, et relativement homogènes dans leurs souhaits en termes de visées morphologiques. 


\section{Les contraintes culturelles}

37 En plus de son action vis-à-vis d'anomalies morphologiques, le discours des sites web analysés définit les interventions qu'il promeut comme novatrices et comme salvatrices en matière d'épanouissement sexuel vis-à-vis de contraintes culturelles. D'une part, il se positionne comme défenseur de la sexualité des femmes en s'opposant à des discours et des pratiques jugées néfastes pour l'épanouissement sexuel relevant de "notre " histoire, qu'il s'agisse de tabous sur la sexualité ou de techniques obsolètes. D'autre part, il présente cette chirurgie comme salvatrice vis-à-vis de pratiques rituelles comprises comme appartenant à d'« autres » cultures. Cette chirurgie n'est donc jamais comprise comme étant une pratique culturelle à la fois locale et contemporaine : elle devient culturelle par référence au passé et à l'ailleurs. Enfin, les organes génitaux masculins ne sont jamais concernés par le lexique renvoyant à la culture, si bien que les pressions culturelles semblent concerner exclusivement les femmes ${ }^{18}$.

Pour évoquer des pratiques locales, le discours réfère à des "tabous» entravant l'épanouissement sexuel des femmes, en recourant à un procédé discursif qui construit une opposition entre un passé encore opérant et l'affirmation d'une posture contemporaine libérale et avertie.

C'est ainsi que les sites affirment et dénoncent à la fois un tabou historique porté sur le sexe, de sorte que le discours qu'ils véhiculent se positionne comme ayant lui-même dépassé le tabou et s'affiche comme un discours de libération :

(31) La région intime est souvent un endroit assez secret du corps et dont on ne parle pas.

(32) À l'heure actuelle, vouloir effectuer une correction esthétique au niveau de la région intime n'est plus un fait exceptionnel.

(33) Il ne se passe pas une semaine sans que je doive expliquer que le vagin est un organe noble et qu'il n'y a rien de honteux à toucher ses organes sexuels.

(34) [D]ans notre société le sujet n'est plus tabou et le droit à l'orgasme est revendiqué haut et fort.

Exploitant l'autorité que leur confère leur inscription dans les sciences médicales, les sites imposent en outre un discours d'expertise en matière d'accès à une sexualité épanouie :

(35) Ces experts veillent à restaurer le plaisir sexuel (libido) du patient et la jeunesse des parties intimes, en remodelant l'intérieur et l'extérieur des organes génitaux.

41 Ce discours promeut des techniques chirurgicales de pointe, capables de remédier, par exemple, aux conséquences de l'épisiotomie :

(36) Durant l'accouchement, c'est dans cette région que l'on pratique l'épisiotomie et que les déchirures dues à l'accouchement sont le plus fréquemment observées. La périnéoplastie (ou périnéorraphie) vise à redonner à cette zone un aspect normal en excisant l'excès de peau et les fibromes mous et en suturant les muscles internes ou le corps périnéal afin de procurer à la patiente une sensation plus agréable dans l'orifice inférieur du vagin. 

anciennes et moins favorables à l'épanouissement sexuel :

(37) La réduction des petites lèvres est longtemps restée un tabou ou décriée même par les gynécologues pratiquant la chirurgie intime. Il est vrai que les premières techniques et même certaines techniques actuelles de réduction de sexe féminin étaient totalement inadaptées. Ces techniques de nymphoplastie, correspondant à une amputation ne doivent plus être pratiquées à l'heure actuelle.

Les chirurgies promues sont non seulement celles qui améliorent la sexualité sur un plan fonctionnel, mais également celles qui ne se voient pas, de sorte que le travail réalisé sur le défaut dont la personne a été affublée ne se remarque pas :

(38) La technique utilisée laisse des cicatrices pratiquement invisibles au point que chez la majorité des opérées il est difficile de voir qu'il y a effectivement eu une intervention.

À la rhétorique de libération procédant par découpage temporel s'associe une division que l'on peut qualifier de coloniale ${ }^{19}$ dans la mesure où elle oppose des traditions oppressives propres à d'autres cultures à la valorisation de l'épanouissement sexuel qui constituerait une caractéristique d'un discours libéral occidental contemporain dont se revendique le discours analysé. Ce dernier propose en effet une série d'interventions visant la réparation d'une morphologie mutilée en renvoyant à un lexique de la culture dans lequel les traces linguistiques sont l'usage de mots comme "rite», "religion", «tradition ». C'est le cas de la réparation du clitoris dans les cas d'excision:

(39) La tradition des «mutilations génitales» touche les femmes des populations originaires de certains pays du continent africain. Leurs séquelles relèvent de souffrances tant physiques que psycho-sexuelles. Les techniques de chirurgie réparatrices existantes permettent de rendre à ces femmes victimes de traditions obsolètes et nuisibles mais encore largement pratiquées ( 2 millions d'excisions par an dans le monde) leur intégrité et leur dignité au prix d'une prise en charge somme toute assez simple.

Là encore, le discours analysé se définit comme défenseur et expert du plaisir sexuel, voire plus encore comme défenseur de "l'intégrité » et «la dignité » des femmes massivement victimes de "traditions ». Cette rhétorique s'illustre également dans une complicité que le discours met en scène vis-à-vis de l'exigence de virginité qui serait propre à certaines «cultures». C'est le cas de l'hyménoplastie, qui construit des hymens à des femmes dont la morphologie empêcherait l'écoulement de sang lors de la nuit de noces ou encore qui permet a posteriori à certaines femmes de vivre une vie sexuelle prémaritale «traditionnellement » prohibée $^{20}$. L'hyménoplastie, lit-on, est :

(40) Pour les femmes appartenant à une culture où l'hymen est le symbole de la virginité et où la tache de sang sur le drap blanc a son importance dans la conclusion d'un mariage (une illusion, car tous les hymens ne saignent pas lors du premier rapport sexuel).

(41) L'institut [X] tient compte des besoins de certaines femmes, liés à l'aspect culturel de leur milieu. En effet, le thème de la virginité, dans certaines cultures, peut être primordial d'un point de vue social, culturel ou religieux. 
Dans des formes contemporaines tout à fait particulières, notamment en raison de la valorisation de l'épanouissement individuel qui l'oriente, ce discours prolonge et réarticule des mécanismes de distinction, voire d'altérisation, entre groupes sociaux qui caractérisent le discours médical sur la morphologie sexuelle dès son émergence. Dans la condamnation de traditions " exotiques " présentées comme particulièrement sexistes, on reconnaît un mécanisme qui a été mis au jour par les sociologues travaillant sur la distinction coloniale entre un Nous et des Autres en fonction du traitement respectif des femmes (Carby 2008; Nader 2006) et qu'Éric Fassin (2006) qualifie d'« impérialisme de la démocratie sexuelle ».

De plus, ces pratiques de modification corporelle relèvent, dans le contexte local et contemporain, de pratiques culturelles. Par exemple, les chirurgies prennent sens en fonction de parcours de vie normatifs, voire ritualisés - l'entrée dans la sexualité à l'adolescence, la maternité, la vieillesse et la sortie du marché sexuel (Bozon 2013) - et elles sont utilisées pour adapter le corps à certaines pratiques vestimentaires et sportives éminemment culturelles (porter des leggings ou des strings, faire du vélo ou de l'équitation).

\section{Mise en perspective}

Comment penser les ressorts de ce discours dans une analyse plus large des représentations du sexe et de la sexualité ? L'analyse du lexique démontre que la chirurgie d'amélioration des organes génitaux est proposée afin de triompher des défauts morphologiques et des contraintes culturelles, ceci en mobilisant la revendication de l'épanouissement sexuel pour les femmes comme pour les hommes, tout en reproduisant une asymétrie de genre. Du point de vue d'une analyse constructiviste, ce discours est paradoxal parce qu'il propose simultanément une libération individuelle face à certaines contraintes sociales, notamment de genre et de culture, tout en reproduisant des rapports de domination et des effets de normalisation. En effet, les modifications proposées répondent à des représentations normées de la sexualité et des identités de sexe. Comprendre cette contradiction apparente exige, de notre point de vue, de tenir compte d'une autre norme sociale, qui est celle de l'injonction à la création de soi.

\section{Triompher des défauts du corps}

Nous l'avons vu, le discours présent sur les sites web étudiés s'adresse d'abord et avant tout aux femmes, en vue d'améliorer leur sexualité supposée contrainte par leur corps et leur destin biologique. Ce faisant, il représente le sexe et la sexualité féminines comme normalement défaillantes : bien qu'une partie du vocabulaire utilisé renvoie au registre de la pathologie, ce discours recourt à des procédés discursifs de banalisation qui définissent les défaillances sexuelles comme ordinaires. Le discours fait donc savoir que les femmes éprouvent diverses détresses dans le cadre de leur sexualité : la chirurgie sexuelle cosmétique corrige les sexes qui sont (devenus) laids et dysfonctionnels parce que ces défauts contrarient l'épanouissement sexuel, voire le bien-être général des femmes. Toutefois, le discours présuppose également que les femmes puissent atteindre l'épanouissement sexuel, adhérant au modèle de la sexualité « libre et sans contrainte » (Bajos, Ferrand \& Andro 2008 : 561) qui s'impose désormais 
comme une norme. Ce discours présente ainsi l'épanouissement sexuel des femmes à la fois comme laborieux et nécessaire.

S'il réfère aux anomalies morphologiques pour expliquer les détresses des femmes, il assure toutefois ne pas capituler devant ces défauts du corps. Les laideurs et usures des organes génitaux féminins ne sont pas fatales du moment que la chirurgie parvient à les annihiler au profit de la création d'organes génitaux jeunes, intérieurs et performants. Cette chirurgie est donc présentée comme l'ennemie des défaillances corporelles, capable de façonner des sexes débarrassés des imperfections individuelles et sortis de la dégénérescence propre à la vie.

51 Le discours analysé ne désigne donc pas ce qui apparaît comme une faillite sexuelle ordinairement programmée des femmes comme nécessaire, ni leurs détresses comme acceptables. En ce sens, il répond aux représentations dominantes de la sexualité, plaidant pour une sexualité non reproductive orientée vers le plaisir et basée sur le partage entre les partenaires (Bozon 2013). Mais, en énumérant des défaillances sexuelles qui limitent les femmes dans leur accès à une telle sexualité, il les fait apparaître, par contraste, comme singulièrement inaptes à une sexualité épanouie, dotées d'« un corps imparfait, faible, malade, impuissant, malsain » (Sagaert $2012: 6$ ).

Lorsque le discours constitue les organes génitaux des femmes comme sujets à des anomalies innées ou acquises sur le parcours de vie, mais qu'il pose comme légitime qu'elles accèdent, malgré cette contrainte, à l'épanouissement sexuel et plus généralement au confort physique, psychologique et relationnel, il s'aligne sur une visée égalitaire, remettant en question la fatalité d'un destin biologique, et il propose des solutions pour dépasser ou plutôt pour déjouer les conséquences de cet irréductible biologique. Autrement dit, avec ce discours, on est loin de l'idée que le sexe impose nécessairement des places et des accès spécifiques aux femmes et aux hommes; mais on est également loin de dénaturaliser les catégories sociales de sexe, les organes génitaux demeurant un critère fondamental et efficient de distinction de sexe. Nous posons l'hypothèse que ce paradoxe est (en partie) résolu par une forme d'individualisation des normes, qui consiste à enjoindre les individus à « devenir soi » en dépit des défauts du corps et des contraintes sociales.

\section{Améliorer ces organes génitaux pour « devenir soi »}

Le discours analysé propose, et non impose, une chirurgie qui permet de «composer une identité de genre " (Macé 2015 : 130) en incorporant des idéaux morphologiques jugés non nécessaires mais qui permettent de se sentir bien dans son sexe et dans sa sexualité. Que les chirurgies doivent être entreprises pour soi-même est une opinion qui sous-tend généralement les discours de la chirurgie esthétique (Fraser 2003 ; Heyes 2007) et qui traduit une individualisation des normes sociales : performer son sexe, en tant que pratique relevant du choix de chacune, devient une question de responsabilité individuelle.

Le discours que nous analysons illustre ainsi le propos de Soulages (2004:56) selon lequel la publicité se présente désormais comme « une idéologie du libre-service, de la liberté de choix où chacun peut être servi ", "ce libre service des désirs et des rêves " n'en illustrant pas moins des « mises en scène des rapports sociaux de domination, qui se dissimulent derrière des jeux incessants de gratification ou d'acculturation ». La chirurgie d'amélioration des organes génitaux est en effet présentée comme une 
technologie du plaisir féminin. Ce faisant, le discours présenté sur les sites web des cliniques et cabinets étudiés redessine ce que Angelof et Gardey (2015 : 33) identifient comme la «ligne de partage entre les techniques reproductives, pensées pour les femmes, et les techniques hédoniques, tel le Viagra, conçues pour les hommes ». Ce recours demeurant optionnel, la production d'une morphologie "bien" sexuée permettant l'incorporation d'une identité féminine optimale (se sentir bien dans son corps, jeune et avec un sexe intériorisé, être sexuellement active et épanouie) passe ainsi pour une question de choix personnel. Dans son analyse des magazines offrant des conseils pour la beauté, Anastasia Meidani $(2014: 187)$ dit la même chose, insistant sur les formes médicalisées de l'esthétique :

De la sorte, les «conseils des professionnels » qui soutiennent l'idée d'une beauté qui se fabrique se répondent et se complètent avec un aura de scientificité qui rime avec l'air de médicalisation du corps. Ainsi avec la caution médicale, l'esthétisme contemporain du "prêt à porter » au "sur-mesure " renforçant l'illusion d'une maîtrise accrue de son corps, une maîtrise "ordonnancée par soi-même ». À ce sujet, la promotion médiatique de la chirurgie esthétique offre des exemples saisissants.

Quels sont les rapports de domination que véhicule le discours que nous étudions? Il s'agit bien sûr d'une part du rapport social de sexe, puisque c'est la physiologie féminine qui, selon lui, contrarie l'accès des femmes à l'épanouissement sexuel. À cet égard, le discours que nous analysons contribue à naturaliser la différence et l'asymétrie des sexes en reconduisant, sous des formes renouvelées, la pathologisation du corps féminin (Oudshoorn 1994), en même temps qu'il se présente comme une solution face à son constat naturaliste. Le discours met également en scène, dans des formes stéréotypées, nous l'avons également vu, un discours évolutionniste et culturaliste: parmi toutes les femmes, certaines sont de plus contrariées par des pratiques oppressives du point de vue de l'épanouissement sexuel. Aussi, vis-à-vis du devoir d'accéder au bien-être, il ne nous semble pas que pas le recours à l'argument soit morphologique soit culturaliste fasse une grande différence : le discours fait surtout savoir que toutes les femmes, pour différentes raisons - leur enculturation, leur âge, leur parcours de vie, leurs caractéristiques innées -, sont potentiellement empêchées d'accéder à une sexualité épanouissante, mais que la responsabilité d'y accéder, ou non, leur revient. Elles sont ainsi rendues responsables de leur faillite sexuelle, quelle qu'en soit la raison :

[U]ne responsabilisation et un autocontrôle, toujours croissants, se font valoir comme le droit de l'individu à disposer de son corps comme il l'entend. Nous discernons aisément le risque d'une tel discours médiatique: la malléabilité physique se confond avec la libération du sujet. Attribuer au corps le statut d'objet susceptible de remaniements incessants, c'est lui renvoyer son insuffisance. (Meidani 2014 : 186)

Il s'agit donc pour les femmes de maîtriser leur sexe - dans tous les sens du terme - et de le mettre au service de leur propre épanouissement. Sur les sites étudiés, la chirurgie d'amélioration des organes génitaux est publicisée comme un moyen mis à disposition des femmes et leur revient la responsabilité d'y recourir pour maitriser une sexualité de plaisir telle qu'elle est valorisée socialement. 


\section{Conclusion}

57 L'analyse du discours de la chirurgie d'amélioration des organes génitaux sur les sites web étudiés montre qu'une partie des caractéristiques des sexes opérables est comprise comme des défaillances normales pouvant affecter, avec d'autres entraves d'ordre culturel, la sexualité et plus généralement la vie des femmes. La diversité morphologique peut bien être reconnue, elle n'en est pas moins traversée par des représentations et des pratiques qui éloignent plus ou moins les groupes d'individus de ce qui est considéré comme bien ou simplement normal. Les femmes telles qu'elles sont définies par le discours étudié forment un groupe qui s'éloigne tendanciellement de la norme de l'épanouissement sexuel. Et, en associant l'évidence des défaillances naturalisées et des pressions culturelles qui contrarient le plaisir sexuel avec la légitimité de l'épanouissement sexuel, le discours étudié définit la chirurgie qu'il promeut comme un instrument de leur libération. Cette libération est une affaire personnelle, puisque se sentir bien, ne pas subir son sexe et sa sexualité, en contrant les effets supposés de sa morphologie et les contraintes culturelles, est un signe de valeur sociale: maîtriser une appartenance genrée nette, jeune et une sexualité épanouie libérée de contraintes relève de la responsabilité de chaque femme.

Pour conclure, revenons sur l'émergence de la chirurgie d'amélioration des organes génitaux. Cette chirurgie, qui se présente comme massivement destinée aux femmes, se construit dans un espace ambigu entre le cosmétique et la réparation, en d'autres termes entre la normalité et la pathologie. Elle renoue, d'une certaine façon, avec les chirurgies correctrices $\mathrm{du} \mathrm{XIX}^{\mathrm{e}}$ siècle: elle intervient sur des organes génitaux considérés comme affectant la sexualité jugée normale, bien qu'elle se déploie dans un contexte technologique engageant à la transformation et l'amélioration de soi. Elle réfère ainsi à des conceptions particulières de la santé, envisagée comme un état de bien-être physique, psychologique et social, et de la sexualité, désormais centrée autour de la performance et du plaisir et considérée comme un signe de valeur individuelle. Ce classement illustre le caractère éminemment social, et historiquement instable, nous l'avons relevé, des frontières entre différentes chirurgies. À cet égard, l'émergence d'un discours et de pratiques de chirurgie d'amélioration des organes génitaux exprime également une faille dans l'évidence de la sexualité de plaisir : en définissant comme ordinaires et comme problématiques des situations de non-accès à une sexualité épanouie, elle met au jour que la sexualité de plaisir n'est pas toujours atteinte et elle participe ainsi à une reconnaissance des difficultés qu'impose le devoir d'épanouissement sexuel. En d'autres termes, dans la perspective qui est la nôtre, l'émergence de ce discours contribue peut-être paradoxalement à mettre au jour les dimensions normatives, contraignantes et arbitraires d'une sexualité de plaisir. 


\section{BIBLIOGRAPHIE}

ANGELOFF, Tania \& GARDEY, Delphine. 2015. « Corps sous emprises. Biopolitique et sexualité au Nord et au Sud » Travail, genre et sociétés 2(34):31-38.

BAJOS, Nathalie, FERRAND, Michèle \& ANDRO, Armelle. 2008. « La Sexualité à l'épreuve de l'égalité » Enquête sur la sexualité en France : pratiques, genre et santé. Paris : La Découverte, 454-576.

BIRKE, Lynda. 1997. « The Black Hole : Women's Studies, Science and Technology », in Introducing Women's Studies, ROBINSON, Victoria \& RICHARDSON, Diane (éds.). London : Macmillan Press.

BOILLOT, Bernard, TEKLALI, Youssef, MOOG, Raphaël \& DROUPY, Stéphane. 2013. « Les Malformations congénitales du pénis » Progrès en urologie [en ligne] 23, consulté le 27 janvier 2016. URL : http://dx.doi.org/10.1016/j.purol.2013.01.022

BOZON, Michel. 2009. « Libération sexuelle ou déplacement des contrôles ? Discours, normes, conduites ", in Reproduire le genre, DORLIN, Elsa \& FASSIN, Éric (éds.). Paris : Bibliothèque du Centre Pompidou, 145-160.

BOZON, Michel. 2013. Sociologie de la sexualité. Paris : Armand Colin.

CARBY, Hazel. 2008 [2000]. « Femme blanche écoute ! Le féminisme noir et les frontières de la sororité », in Black feminism. Anthologie du féminisme africain-américain, 1975-2000, DORLIN, Elsa (éd.). Paris : L’Harmattan, 87-112.

CHAPERON, Sylvie. 2002. «L'Histoire des sexualités en France » Vingtième siècle. Revue d'histoire $75: 47-59$.

CHAPERON, SYLVIE. 2004. « Contester normes et savoirs sur la sexualité (France-Angleterre, 1880-1980) », in Le siècle des féminismes, GUBIN, Eliane, JACQUES, Catherine, ROCHEFORT, Florence, STUDER, Brigitte, THÉBAUD, Françoise \& ZANCARINI-FOURNEL, Michelle (éds.). Paris : Les Éditions de l'Atelier / Éditions Ouvrières, 333-346.

CHAPERON, Sylvie. 2008. La Médecine du sexe et les femmes : anthologie des perversions féminines au XIX $X^{e}$ siècle. Paris : La Musardine.

CHAPERON, Sylvie. 2010. « Du Féminisme à la sexologie : un parcours en Histoire » Genre, sexualité et société [en ligne] 4, consulté le 26 août 2016. URL : http://gss.revues.org/index1672.html DAVIS, Kathy. 1995. Reshaping the Female Body : the Dilemma of Cosmetic Surgery. London : Routledge.

DAVIS, Kathy. 2002. «A Dubious Equality : Men, Women and Cosmetic Surgery » Body \& Society 49 : 35-55.

DELPHY, Christine. 2001. « Penser le genre : problèmes et résistances », in L'Ennemi principal 2. Penser le genre, DELPHY, Christine (éd.). Paris : Syllepse, 243-260.

ERLICH, Michel. 2007. « La Chirurgie sexuelle en France : aspects historiques » Sexologies [en ligne] 16, consulté le 13 janvier 2016. URL : http://www.sciencedirect.com/science/article/pii/ S1158136007000953

FASSIN, Éric. 2006. « La Démocratie sexuelle et le conflit de civilisation » Multitudes 26 : 123-131.

FAUSTO-STERLING, Anne \& TOURAILLE, Priscille. 2014. « Autour des critiques du concept de sexe. Entretien avec Anne Fausto-Sterling » Genre, sexualité \& société [en ligne] 12, consulté le 2 mars 2015. URL : http://gss.revues.org/3290 
FAUSTO-STERLING, Anne. 1999. Sexing the body: Gender politics and the Construction of Sexuality. New York : Basic Book.

FOLDÈS, Pierre, DROUPY, Stéphane \& CUZIN, Béatrice. 2013. « Chirurgie cosmétique de l'appareil génital féminin » Progrès en urologie [en ligne] 23, consulté le 27 janvier 2016. URL : http:// dx.doi.org/10.1016/j.purol.2013.01.017

FOUCAULT, Michel. 1976. Histoire de la sexualité 1. La Volonté de savoir. Paris : Gallimard.

FRASER, Suzanne. 2003. «The Agent Within : Agency Repertoires in Medical Discourse on Cosmetic Surgery » Australian Feminist Studies $40: 27-44$.

GARDEY, Delphine. 2013. « Comment écrire l'histoire des relations corps, genre, médecine au xx siècle?»Clio. Quand la médecine fait le genre 37 : 143-162.

GARDEY, Delphine \& HASDEU, Iulia. 2015. «Cet obscur sujet du désir. Médicaliser les troubles de la sexualité féminine en Occident » Travail, genre et sociétés 34 : 73-89.

GIAMI, Alain. 2007. « Santé sexuelle : la médicalisation de la sexualité et du bien-être » Le Journal des Psychologues [en ligne] 7, consulté le 15 février 2016. URL : http://www.cairn.info/revue-lejournal-des-psychologues-2007-7-page-56.htm

GILMAN, Sander L. 1985. « Black Bodies, White Bodies : Toward an Iconography of Female Sexuality in Late Nineteenth-Century Art » Medicine, and Literature. Critical Inquiry [en ligne] 12, consulté le 15 février 2016. URL : http://www.jstor.org/stable/1343468

GILMAN, Sander L. 1997. « Decircumcision : The First Aesthetic Surgery » Modern Judaism [en ligne] 3, consulté le 17 mars 2015. URL : http://www.jstor.org/stable/1396657?

seq=1\#page_scan_tab_contents

GOODMAN, Michael P. 2009. « Female Cosmetic Genital Surgery » Obstetrics \& Gynecology 113 : 154-159.

GRINO, Claire. 2014. « Prescrire un contraceptif : le rôle de l'institution médicale dans la construction de catégories sexuées » Genre, sexualité \& société [en ligne] 12, consulté le 15 août 2015. URL : http://gss.revues.org/3280

GUIRIMAND, Nicolas. 2005. "De la réparation des "gueules cassées" à la "sculpture du visage" " Actes de la recherche en sciences sociales [en ligne] 1, consulté le 18 janvier 2016. URL : http:// www.cairn.info/revue-actes-de-la-recherche-en-sciences-sociales-2005-1-page-72.htm HALBERSTAM, Judith. 1998. Female Masculinity. Durham and London : Duke University Press.

HARAWAY, Donna. 1988. « Situated Knowledges : The Science Question in Feminism as a Site of Discourse of the Privilege of Partial Perspective » Feminist Studies 14(3) : 575-599.

HARAWAY, Donna. 1991. Simians, Cyborgs, and Women. The Reinvention of Nature. London : Free Association Books.

HARDING, SANDRA. 1991. Whose Science? Whose's Knowlegde? Thinking from Women's Lives. Ithaca, New York : Cornell University Press.

HEYES, Cressida J. 2007. « Cosmetic Surgery And The Televisual Makeover » Feminist Media Studies [en ligne] 1, consulté le 14 janvier 2015. URL : http://www.tandfonline.com/doi/abs/ 10.1080/14680770601103670\#.V06psTf2FFQ

HODGKINSON, Darryl J. \& HAIT, Glen. 1984. « Aesthetic Vaginal Labiaplasty » Plastic Reconstructive Surgery $74(3)$ : 414-416. 
HOGLE, Linda F. 2005. « Enhancement Technologies and the Body » Annual review of anthropology [en ligne] 34, consulté le 10 novembre 2015. URL : http://www.annualreviews.org/doi/abs/ 10.1146/annurev.anthro.33.070203.144020

JAUNAIT, Alexandre, RAZ, Michel, \& RODRIGUEZ, Eva. 2014. « La biologisation de quoi ? » Genre, sexualité \& société [en ligne] 12, consulté le 18 août 2016. URL : http://gss.revues.org/3317

KERGOAT, Danièle. 2009. "Dynamique et consubstantialité des rapports sociaux ", in Sexe, race, classe, pour une épistémologie de la domination, DORLIN, Elsa (éd.). Paris : PUF, 111-125.

LE HÉNAFF, Yannick. 2013. «Catégorisations professionnelles des demandes masculines de chirurgie esthétique et transformations politiques de la médecine » Sciences sociales et santé 3(31): 39-64.

LÖWY, Ilana. 2003. « Intersexe et transsexualités : les techniques de la médecine et la séparation du sexe biologique du sexe social » Cahiers du Genre 34 : 81-104.

MACÉ, Éric. 2010. «Ce que les normes de genre font au corps, ce que les corps trans font aux normes de genre » Sociologie 4 : 497-515.

MACÉ, Éric. 2015. L’Après-patriarcat. Paris : Seuil.

MARTIN, Hélène. 2014. « Le Beau Sexe. Quelques réflexions sur les chirurgies sexuelles cosmétiques » Genre, sexualité \& société [en ligne] 12, consulté le 28 août 2016. URL : https:// gss.revues.org/3222

MARTIN, Hélène, HERTZ, Ellen \& REY, Séverine. 2015. « Une Disgrâce commune. Pour une anthropologie symétrique des pratiques de marquage du sexe », in Mélanges en l'honneur de Mondher Kilani, CERQUI, Daniela \& MAFFI, Irene (éds.). Lausanne : BSN Press, 105-123.

MEIDANI, Anastasia. 2014. «Corps, genre et magazines de presse. Dimorphisme physiologique et dimorphisme moral : un corps pour chacun? ", in Quand la médiatisation fait genre. Médias, transgression et négociations de genre, BISCARRAT, Laetitia, ESPINEIRA, Karine, THOMAS, MaudYeuse, ALESSANDRIN, Arnaud (éds.). Paris : L'Harmattan, 179-194.

NADER, Laura. 2006. « Orientalisme, occidentalisme et contrôle des femmes » Nouvelles Questions Féministes $1: 12-24$.

NURKA, Camille \& Jones, Bethany. 2013. « Labiaplasty, Race and the Colonial Imagination » Australian Feminist Studies 28(78) : 417-442.

OFS (Office fédéral de la statistique). 2015. Indicateur des médias - Internet - Utilisation d'Internet, consulté le 2 avril 2015. URL : http://www.bfs.admin.ch/bfs/portal/fr/index/themen/16/03/ key/ind16.indicator.30106.160204.html

OUDSHOORN, Nelly. 1994. Beyond the Natural Body : An Archeology of Sex Hormones. London : Routledge.

PAHUD, Stéphanie. 2006. «Circulation publicitaire des discours sur les sexes » Tranel (Travaux neuchâtelois de linguistique) [en ligne] 44, consulté le 7 avril 2015. URL : http://doc.rero.ch/record/ 17522

PEIRETTI-COURTIS, Delphine. 2015. «Sexe, race et médecine. Anatomie et sexualité des Africain·e's sous l'œil des médecins français (1780-1950) » Émulations [en ligne] 15, consulté le 27 janvier 2016. URL : https://ojs.uclouvain.be/index.php/emulations/article/view/4963

PERROT, Michelle. 1998. "Michel Foucault et l'histoire des femmes ", in Les Femmes ou le silence de l'Histoire, PERROT, Michelle (éd.). Paris : Flammarion, 413-424. 
PRECIADO, Paul (Beatriz). 2005. «Biopolitique du genre », in Le corps, entre sexe et genre, ROUCH, Hélène, DORLIN, Elsa \& FOUGEYROLLAS-SCHWEBEL, Dominique (éds.). Paris : L'Harmattan, 61-84. QUEVAUVILLIERS, Jacques, SOMOGYI, Alexandre \& FINGERHUT, Abe (éd.). 2009 [6 éd.]. Dictionnaire médical. Issy-les-Moulineaux : Elsevier Masson.

QUIJANO, Anibal. 2007. «"Race" et colonialité du pouvoir » Mouvements 3(51) : 11-118.

SACRISTE, Valérie. 2001. « Sociologie de la communication publicitaire » L'Année sociologique [en ligne] 2, consulté le 6 juin 2015. URL : http://www.cairn.info/revue-l-annee-sociologique-2001-2page-487.htm

PFEFFERKORN, Roland. 2012. Genre et rapports sociaux de sexe. Lausanne : Éditions Page 2.

SAGAERT, Claudine. 2012. « Les Fondements ontologiques de la laideur féminine » Revue Horizon Sociologique [en ligne] Hors-Champ, consulté le 05 mai 2015. URL : http://www.revuesociologique.org/node/13438

SAID, Edward. 2000. Culture et impérialisme. Paris : Fayard et Le Monde diplomatique.

SCHIEBINGER, Londa. 2004. Nature's body. Gender and the Making of Modern Science. New Brunswick : Rutgers University Press.

SCULL, Andrew \& FAVREAU, Diane. 1987. « Médecine de la folie ou folie des médecins : controverse à propos de la chirurgie sexuelle au XIX ${ }^{\mathrm{e}}$ siècle » Actes de la recherche en sciences sociales [en ligne] 68, consulté le 31 mai 2016. URL : http://www.persee.fr/doc/ arss_0335-5322_1987_num_68_1_2372

SOULAGES, Jean-Claude. 2004. «Le genre en publicité, ou le culte des apparences » MEI « Médiation et information » [en ligne] 20, consulté le 7 avril 2015. URL : http://www.mei-info.com/wpcontent/uploads/revue20/ilovepdf.com_split_6.pdf

TARAUD, Christelle. 2011. " La virilité en situation coloniale », in Histoire de la virilité 2. Le triomphe de la virilité. Le XIX ${ }^{e}$ siècle, CORBIN, Alain (éd.). Paris : Seuil, 331-347.

TIEFER, Leonor. 2008. « Female Genital Cosmetic Surgery : Freakish or Inevitable? Analysis from Medical Marketing, Bioethics, and Feminist Theory » Feminism and Psychology [en ligne] 18, consulté le 9 octobre 2014. URL : http://fap.sagepub.com/content/18/4/466

VIGARELLO, Georges. 2004. Histoire de la beauté. Le corps et l'art d'embellir de la Renaissance à nos jours. Paris : Seuil.

VUILLE, Marilène. 2014. «Le Désir sexuel des femmes, du DSM à la nouvelle médecine sexuelle » Genre, sexualité \& société [en ligne] 12, consulté le 20 mai 2016. URL : http://gss.revues.org/3240

WOLF, Naomi. 1990. The Beauty Myth : How Images of Beauty Are Used Against Women. London : Chatto \& Windus.

Corpus numérique

www.aesthetics-ge.ch (dernière consultation le 29.05.2015) ;

www.centrefeelwell.ch (dernière consultation le 29.05.2015) : (21), (28) ;

www.chirurgie-plastique-geneve.ch (dernière consultation le 29.05.2015) : (1), (25), (30), (39) ;

www.delc.ch (dernière consultation le 29.05.2015) : (4), (6), (31), (32) ;

www.dr-heer.ch (dernière consultation le 29.05.2015, ce site n'est plus consultable au 5.12.2016) : (2), (5), (12), (13), (14), (18), (20), (26), (41) ;

www.laprairie.ch (dernière consultation le 29.05.2015) : (8), (15), (23), (35), (36) ; 
www.medfemme.com (dernière consultation le 29.05.2015) : (7), (16), (19), (24), (27), (33), (34),

$(40)$;

www.nymphoplastie.fr (dernière consultation le 29.05.2015) : (37)

www.olivierbauquis.ch (dernière consultation le 29.05.2015)

www.riviera-clinic.ch (dernière consultation le 29.05.2015) : (3), (9), (10), (11), (17), (22), (29) ;

www.swisswomensclinic.com (dernière consultation le 29.05.2015) : (38).

\section{NOTES}

1. http://www.laprairie.ch/fr/pôles-de-compétences/centre-de-médecine-et-chirurgieesthétique (consulté le 24 août 2016)

2. Intitulée "Chirurgie sexuelle cosmétique: quelles représentations du corps sexué? Une approche en étude genre ", la recherche est financée par le Fonds national suisse de la recherche scientifique, division 1 (sciences humaines et sociales). Les autres corpus sont constitués par des entretiens menés avec des professionnelles (à ce jour, dix-sept spécialistes en gynécologie et en chirurgie), des forums de discussion et des articles de presse (une trentaine de discussions sur les forums Au Féminin et Doctissimo et des articles de la presse suisse romande parus entre 2003 et 2016). Nous y faisons brièvement référence lorsque cela s'avère intéressant dans le cadre de cet article.

3. Il s'agit d'une théorie, dite transformiste, selon laquelle la fonction développe l'organe alors que l'inaction entraîne son atrophie.

4. En témoigne la définition actuelle de l'OMS: «La santé est un état de complet bien-être physique, mental et social, et ne consiste pas seulement en une absence de maladie ou d'infirmité. » http://www.who.int/about/definition/fr/print.html (consulté le 17 février 2016)

5. À partir des années 1980, des sexologues revisitent le cycle normal de la sexualité et le sexuent (Vuille 2014); le modèle fonctionnaliste cède la place à une recherche orientée par les neurosciences qui, accompagnée par l'industrie pharmaceutique, produit de nouvelles catégories de troubles de la sexualité et des traitements en conséquence (Gardey \& Hasdeu 2015).

6. Ce concept résulte en effet « de la rencontre entre deux domaines théorico-pratiques [la santé et la sexualité] qui se sont modernisés et qui ont placé la question du bien-être, de son accompagnement, de son encadrement et de son développement, au centre de leurs préoccupations et de leurs interventions » (Giami $2007: 56$ ).

7. Les technologies de (ré)assignation sexuelle mais aussi toutes celles qui se sont développées dans le cadre du développement de la gynécologie, de l'obstétrique et de l'urologie.

8. Il s'agit de Hodgkinson, Darryl J. \& Hait, Glen. 1984. « Aesthetic vaginal labiaplasty » Plastic Reconstructive Surgery 74(3) : 414-416.

9. Lors des entretiens, la question était posée aux spécialistes qui publicisent leurs interventions sur des sites web. En synthèse, nous pouvons constater que sur dix-sept entretiens de médecins, huit décident au moins partiellement du contenu de leur site. Pour quatre médecins, nous n'avons pas l'information sur la décision du contenu sur les sites. Enfin, cinq entretiens ont été réalisés avec des médecins travaillant en hôpital et qui n'ont pas de site web promouvant ces chirurgies esthétiques des organes génitaux.

10. Cette qualification est spécifique à ce corpus particulier, ne comprenant pas l'entier du discours " publicitaire » consacré à cette chirurgie (comme les brochures d'information mises à disposition dans certains cabinets et cliniques dans leur salle d'attente, ni encore des vidéos qui sont présentées sur les sites ou ailleurs sur le web). 
11. Article 20, p. 8 et annexe 2, p. 2 du Code de déontologie de la FMH (entrée en vigueur le $1^{\text {er }}$ juillet 1997 et dernière modification réalisée le 28 avril 2016).

12. Notre corpus est donc limité dans le temps. Nous référons dans ce texte aux sites que nous avons étudiés mais ces derniers sont susceptibles de changer, voire d'être supprimés. Les changements sur le web étant inhérents aux corpus numériques, nous ne sommes pas en mesure de fournir les adresses valides de toutes les pages étudiées, certaines ayant disparu. Notre méthodologie repose en effet sur un matériel limité dans le temps. Les adresses valides fournies dans l'article permettent cependant au lectorat de se faire une idée du type de ressources analysées.

13. «Gender dysphoria », DSM-5, 2013, publié par l'American Psychiatric Association.

14. http://www.chuv.ch/dedop/dedop-endo-variations-du-developpement-sexuel_.htm (consulté le 23 mai 2016)

15. http://www.olivierbauquis.ch/soins.php?traitement=4\&article=16 (consulté le 23 mai 2016)

16. http://www.chuv.ch/cpr/cpr_home/cpr-patients-familles/cpr-chirurgie_transgenre.htm (consulté le 23 mai 2016)

17. Épisiotomie : «Incision chirurgicale du périnée, le plus souvent oblique, en partant de la commissure postérieure de la vulve afin d'éviter une déchirure grave du périnée au cours de l'accouchement et de faciliter la sortie du fœetus » (Quevauvilliers et al. 2009 : 328).

18. En revanche, la littérature montre que la circoncision fait l'objet de polémiques, hier comme aujourd'hui (Erlich 2007 ; Gilman 1997). Par exemple, dans un contexte européen marqué par l'antisémitisme, la reconstruction du prépuce, qui apparaît aux XVIII ${ }^{\mathrm{e}}$ et $\mathrm{XIX}^{\mathrm{e}}$ siècles en Allemagne et en Autriche, est pratiquée dans le but d'effacer une marque d'altérité qui associe à une morphologie des défauts moraux tels que l'efféminement, la laideur et l'absence de santé. Sur les forums de discussion que nous analysons par ailleurs, elle est l'objet de questionnements inscrits dans la valorisation de l'épanouissement sexuel: on débat de la question de la diminution de plaisir qu'elle pourrait ou non engendrer.

19. Nous entendons par "division coloniale" une distinction issue d'un rapport social qui organise une séparation hiérarchisée du monde entre l'Occident et les Autres; ce couple peut être qualifié différemment au cours du temps (civilisé / sauvage, évolué / primitif, moderne / traditionnel, développé / en développement, etc.), mobilisé dans différents registres et contextes (Carby 2008), mais la logique d'opposition et d'asymétrie demeure (Quijano 2007 ; Said 2000).

20. À cet égard, les entretiens menés avec des chirurgiens sont intéressants : cette " complicité » fait l'objet de positionnements sensiblement différents ou du moins de pondération de la part de certains médecins : ils se demandent si réaliser des hyménoplasties est légitime, dans la mesure où cela pourrait reconduire une tradition qu'ils réprouvent. Certains médecins réfèrent à l'argument du risque couru pour justifier leurs interventions, un risque qu'ils évaluent donc euxmêmes : plus le risque pour la femme est grand, plus la modification est légitime.

\section{RÉSUMÉS}

Cet article propose une analyse du discours des sites web de cliniques et cabinets médicaux de Suisse romande qui promeuvent des chirurgies d'amélioration des organes génitaux. Il a pour objectif de comprendre comment ce discours représente les organes génitaux et la sexualité, en portant une attention particulière à l'articulation du biologique et du social et aux rapports de 
domination. Au moyen d'une analyse basée sur le repérage de procédés discursifs et de champs lexicaux, il met au jour trois thèmes qui constituent les ressorts du discours analysé: la promotion de l'épanouissement sexuel et deux types d'obstacles qui entraveraient l'épanouissement sexuel des femmes. Ces obstacles sont des anomalies morphologiques innées ou acquises sur le parcours de vie d'une part et, d'autre part, des discours et des pratiques jugées culturelles et oppressives. Alors que les organes génitaux féminins associés à la sexualité apparaissent comme tendanciellement défaillants, les organes génitaux masculins associés à la sexualité, peu évoqués sur les sites, paraissent morphologiquement appropriés à la sexualité et affranchis de contraintes culturelles. L'article développe l'idée que les rapports sociaux de sexe et de culture qui ressortent de l'analyse s'articulent avec une individualisation des normes qui passe par l'injonction à « devenir soi ».

This article explores and analyses the discourse presented on clinics' and doctor's offices' websites that promote genital improvement surgeries in French-speaking Switzerland. Paying particular attention to power relations and to the articulation of the biological and the social, it aims at understanding how genitals and sexuality are presented. Using an analysis based on the identification of discursive processes and lexical fields, the authors identify three main themes : the promotion of sexual fulfillment and two types of obstacles understood as preventing its achievement. These obstacles are, on the one hand, innate or acquired morphological abnormalities and, on the other hand, discourses and practices considered oppressive and of a 'cultural' nature. The article develops the idea that, through the imperative to 'become our true selves', gender, culture and class intersect with the process of individualization of social norms.

\section{INDEX}

Keywords : genital surgery, web sites, sexuality, gender, culture, production of self Thèmes : Recherches

Mots-clés : chirurgie des organes génitaux, sites web promotionnels, sexualité, production de soi, genre, culture

\section{AUTEURS}

\section{HÉLÈNE MARTIN}

Haute école de travail social et de la santé (HES·SO), Laboratoire de recherche santé-social (LaReSs).

Hélène Martin est docteure en anthropologie, professeure ordinaire à la Haute école spécialisée de travail social et de la santé (HES·SO) où elle est co-responsable du Réseau Genre et Travail social (GeTS). Elle dispense des cours en études genre aux niveaux Bachelor et Master et mène des recherches dans les domaines de la sexualité et de l'imbrication des rapports sociaux. Parmi ses dernières publications : « Le beau sexe. Quelques pistes de réflexion sur les chirurgies sexuelles cosmétiques » Genre, sexualité \& société 12, 2014 [http://gss.revues.org/3222] ; avec Patricia Roux, «Imbrication des rapports de pouvoir » Nouvelles Questions Féministes 34(1) [https://www.cairn.info/revue-nouvelles-questions-feministes-2015-1.htm]. Lausanne : Antipodes, 2015. 


\section{REBECCA BENDJAMA}

Haute école spécialisée de travail social et de la santé (HES·SO), université de Neuchâtel.

Rebecca Bendjama est chargée de recherche à la Haute école spécialisée de travail social et de la santé (HES-SO) et doctorante à l'Université de Neuchâtel. Son travail de thèse porte sur le discours déconstructionniste dans la presse féministe non académique en Suisse romande. Elle a publié, avec Denis Mieville, «Les "filles" “préfèrent" le "rose" : un exemple d'activité de déconstruction dans l'émiliE » TrajEthos 1(1), 21-35, 2012.

\section{RAPHAËLLE BESSETTE-VIENS}

Haute école spécialisée de travail social et de la santé (HES·SO).

Raphaëlle Bessette-Viens a obtenu une maîtrise en études genre de l'Université de Genève en 2015. Elle est engagée depuis 2014 en tant que chargée de recherche à la Haute école de travail social et de la santé de Lausanne (HES·SO). Sur la base de son mémoire de Master, elle a publié : «Mouvements féministes en Suisse francophone » Féminétudes, Héritages féministes : tensions et parallèles 20, 66-73, 2015. 